\title{
Editorial
}

Published online: December 11, 2017

DOI: $10.1159 / 000484669$

\section{Will Canagliflozin Lend Credence to the Potential Effects of Sodium-Glucose Co-Transporter 2 Inhibitors on Renal Endpoints in Diabetic Nephropathy?}

\author{
Joshua J. Neumiller ${ }^{a}$ Connie M. Rhee ${ }^{b}$ Kamyar Kalantar-Zadeh ${ }^{b}$ \\ a Department of Pharmacotherapy, College of Pharmacy, Washington State University, Spokane, WA, USA; \\ ${ }^{b}$ Harold Simmons Center for Chronic Disease Research and Epidemiology, University of California Irvine, \\ School of Medicine, Orange, CA, USA
}

Diabetic kidney disease (DKD) is a major, yet underappreciated, contributor to the global burden of disease [1]. Globally, an estimated 415 million people lived with diabetes in 2015, with the worldwide prevalence of diabetes projected to increase to 642 million in 2040 if current trends continue [1]. With an increase in the prevalence of diabetes comes an increase in the incidence of DKD. While improvements in diabetes management have translated to meaningful improvements in many diabetes-related outcomes, its impact upon DKD and the development of end-stage renal disease (ESRD) have been less robust [2]. In fact, it has been shown that the majority of the excess risk for all-cause and cardiovascular disease (CVD)-related mortality in people with diabetes is related to the presence of DKD [3]. Given the undeniable impact of DKD, there are clear health and economic justifications for improving the outcomes of people living with this chronic disease.

The sodium-glucose co-transporter 2 (SGLT-2) inhibitor class of medications have received considerable recent attention as related to their impact on CVD and renal outcomes - largely driven by findings reported from two key cardiovascular outcome trials (CVOTs): The Empagliflozin Cardiovascular Outcomes and Mortality in Type 2 Diabetes (EMPA-REG OUTCOME) trial $[4,5]$ and the Canagliflozin Cardiovascular Assessment Study (CANVAS) [6]. The EMPA-REG OUTCOME clinical trial was the first CVOT performed with an antihyperglycemic agent to report statistically significant reductions in CVD and renal outcomes [4, 5]. Empaglifozin significantly lowered rates of death from CV causes (4 vs. 6\%; $38 \%$ relative risk reduction), hospitalization for heart failure ( 3 vs. $4 \% ; 35 \%$ relative risk reduction), and death from any cause ( 6 vs. $8 \% ; 32 \%$ relative risk reduction) in patients with type 2 diabetes mellitus and baseline CVD [4]. Post hoc analyses of the EMPA-REG trial also showed beneficial renal effects including a reduction of albuminuria, slowing down of estimated glomerular filtration rate (eGFR) decline, and a 50\% reduction in risk of progressing to ESRD [5]. Similar to EMPA-REG OUTCOME, CANVAS reported significantly lower rates for the primary outcome (composite of death from CV causes, non-

\section{KARGER}

(c) 2017 S. Karger AG, Basel

E-Mail karger@karger.com

www.karger.com/ajn
Joshua J. Neumiller, PharmD, CDE

Department of Pharmacotherapy, College of Pharmacy

Washington State University, PO Box 1495

Spokane, WA 99005 (USA)

E-Mail jneumiller@wsu.edu 
Table 1. Dosing recommendations for sodium-glucose co-transporter 2 inhibitor use in diabetic kidney disease

\begin{tabular}{|c|c|}
\hline Agent & Dosing recommendations based on eGFR, $\mathrm{mL} / \mathrm{min} / 1.73 \mathrm{~m}^{2}$ \\
\hline Empagliflozin & $\begin{array}{l}\text { No dose adjustment required if eGFR } \geq 45 \\
\text { Avoid use and discontinue in patients with eGFR persistently }<45\end{array}$ \\
\hline Canagliflozin & $\begin{array}{l}\text { No dose adjustment required if eGFR } \geq 60 \\
100 \text { mg daily if eGFR } 45-59 \\
\text { Avoid use and discontinue in patients with eGFR persistently }<45\end{array}$ \\
\hline Dapagliflozin & $\begin{array}{l}\text { Avoid initiating if eGFR }<60 \\
\text { Not recommended with eGFR } 30-60 \\
\text { Contraindicated with eGFR }<30\end{array}$ \\
\hline
\end{tabular}

fatal myocardial infarction, or nonfatal stroke) in participants receiving canagliflozin versus placebo (HR 0.86, 95\% CI 0.75-0.97; $p<0.001$ for noninferiority; $p=0.02$ for superiority) [6]. In regard to renal outcomes, the CANVAS investigators noted that on the basis of prespecified hypothesis sequence, the designated renal outcomes reported for the trial were not viewed as statistically significant [6]. Nonetheless, CANVAS reported "possible benefits" of canagliflozin treatment for both progression of albuminuria (HR 0.73, 95\% CI 0.67-0.79) and on the composite renal outcome of a sustained $40 \%$ reduction in eGFR, the need for renal-replacement therapy, or death from renal causes (HR 0.60, 95\% CI 0.47$0.77)$. Indeed, these findings with canagliflozin are further supported by a post hoc analysis from the CANTATA-SU trial that showed canagliflozin treatment, when compared to the addition of glimepiride in patients on background metformin, significantly decreased albuminuria and slowed down eGFR decline [7].

While these findings are both promising and exciting, and in the case of EMPA-REG OUTCOME, have resulted in major changes to clinical treatment recommendations from such organizations as the American Diabetes Association, questions stillabound regarding the mechanism(s) of renal protection, identification of patients most likely to benefit from SGLT-2 inhibitor therapy, and the risk/ benefit balance for SGLT-2 inhibitor use in people with DKD. While the weight and blood pressure benefits of SGLT-2 inhibitors largely persist in patients with DKD (at least in patients with stage 3 chronic kidney disease $[\mathrm{CKD}]$ ), the glucose-lowering benefits are diminished with a decline in eGFR. For this reason, at least in part, current SGLT-2 inhibitor labeling recommends dose reductions based on eGFR, with use not recommended for empagliflozin and canagliflozin in patients with an eGFR $<45 \mathrm{~mL} / \mathrm{min} / 1.73 \mathrm{~m}^{2}$, and use of dapagliflozin contraindicated in patients with an eGFR $<30 \mathrm{~mL} / \mathrm{min} / 1.73 \mathrm{~m}^{2}$ (Table 1).

Fortunately, additional work is being done to shed more light on such unanswered questions. In this issue of the American Journal of Nephrology, Jardine et al. [8] present the study rationale and design for the much anticipated Canagliflozin and Renal Endpoints in Diabetes and Established Nephropathy Clinical Evaluation (CREDENCE) study. CREDENCE is a randomized, double-blind, placebo-controlled, parallel-group, event-driven multicenter study of the effects of canagliflozin, a SGLT-2 inhibitor, on renal and cardiovascular outcomes in subjects with type 2 diabetes mellitus and diabetic nephropathy with eGFRs $\geq 30-90 \mathrm{~mL} / \mathrm{min} / 1.73 \mathrm{~m}^{2}$ and albuminuria (urinary albumin-to-creatinine ratio $>300$ to $\leq 5,000 \mathrm{mg} / \mathrm{g}$ ). The primary outcome measure for CREDENCE is a composite endpoint that includes ESRD, doubling of serum creatinine, and renal or CV death. Given the paucity of available pharmacotherapies that ameliorate the decline of DKD, the CREDENCE trial has the potential to substantially impact the fields of nephrology and endocrinology. As noted by Jardine et al. [8], CREDENCE, due to unique aspects of the population enrolled and the design of the study, will provide important data that will add to the knowledge gained from the CANVAS and EMPA-REG OUTCOME studies. Notable unique aspects of the trial include (1) its use of CKD-EPI for ascertainment of kidney function; (2) inclusion of patients at the highest risk for kidney disease progression (proteinuric CKD patients); (3) its prerequisite of participants receiving a stable regimen of the maximum tolerated dose of an angiotensin-converting enzyme inhibitor or angiotensin receptor blocker; (4) and its prima- 
ry composite outcome that incorporates both renal and CVD endpoints. In terms of safety analyses, CREDENCE will also provide additional insight into the finding of increased amputation risk that was reported in CANVAS [6].

Recently reported cardiovascular and renal outcome data from agents within the SGLT-2 inhibitor class are nothing short of exciting. Additional data from CREDENCE and other pending renal/CVOTs will enhance our collective knowledge and begin to inform some of the unanswered questions pertaining to SGLT-2 in- hibitor use in patients with $\mathrm{DKD}$, and will hopefully propel us closer to "moving the needle" on renal and CVD outcomes in the DKD population.

\section{Disclosure Statement}

Dr. Kamyar Kalantar-Zadeh has received honoraria and/or support from Abbott, Abbvie, Alexion, Amgen, Astra-Zeneca, Aveo, Chugai, DaVita, Fresenius, Genentech, Haymarket Media, Hospira, Kabi, Keryx, Novartis, Pfizer, Relypsa, Resverlogix, Sandoz, Sanofi, Shire, Vifor, UpToDate, ZS-Pharma.

\section{References}

1 Alicic RZ, Rooney MT, Tuttle KR: Diabetic kidney disease: challenges, progress, and possibilities. Clin J Am Soc Nephrol 2017;18:pii: CJN.11491116.

${ }_{2}$ Gregg EW, Li Y, Wang J, Burrows NR, Ali MK, Rolka D, Williams DE, Geiss L: Changes in diabetes-related complications in the United States, 1990-2010. N Engl J Med 2014; 370:1514-1523.

3 Afkarian M, Sachs MC, Kestenbaum B, Hirsch IB, Tuttle KR, Himmelfarb J, de Boer IH: Kidney disease and increased mortality risk in type 2 diabetes. J Am Soc Nephrol 2013;24:302-308.
Zinman B, Wanner C, Lachin JM, et al; EM PA-REG OUTCOME Investigators: Empagliflozin, cardiovascular outcomes, and mortality in type 2 diabetes. N Engl J Med 2015; 373:2117-2128

Wanner C, Inzucchi SE, Lachin JM, et al; EMPA-REG OUTCOME Investigators: Empagliflozin and progression of kidney disease in type 2 diabetes. N Engl J Med 2016;375:323334.

Neal B, Perkovic V, Mahaffey KW, et al; CANVAS Program Collaborative Group: Canagliflozin and cardiovascular and renal events in type 2 diabetes. N Engl J Med 2017; 377:644-657.
Heerspink HJ, Desai M, Jardine M, Balis D, Meininger G, Perkovic V: Canagliflozin slows progression of renal function decline independently of glycemic effects. J Am Soc Nephrol 2017;28:368-375.

Jardine MJ, Mahaffey KW, Neal B, Agarwal R, Bakris GL, Brenner BM, Bull S, Cannon CP, Charytan DM, de Zeeuw D, Edwards R, Greene T, Heerspink HJL, Levin A, Pollock C, Wheeler DC, Xie J, Zhang H, Zinman B, Desai $\mathrm{M}$, Perkovic V: The canagliflozin and renal endpoints in diabetes with established nephropathy clinical evaluation (CREDENCE) study rationale, design and baseline characteristics. Am J Nephrol 2017;46:462-472. 